> Les anticorps radiomarqués ont maintenant fait leurs preuves dans le traitement des lymphomes malins non hodgkiniens de phénotype lymphocytaire B. D'autres maladies cancéreuses s'avèrent plus difficiles à traiter, que les cellules soient trop dispersées pour un traitement avec un émetteur de particules $\beta^{-}$peu efficaces contre des cellules isolées (cas des leucémies par exemple) ou que ces cellules, comme dans la plupart des tumeurs solides, soient radiorésistantes. Des approches nouvelles, préciblage, utilisation d'atomes émetteurs de particules $\alpha$ ou d'électrons Auger sont en passe d'apporter des solutions convaincantes et la radio-immunothérapie devrait trouver sa place dans une approche rationnelle du traitement des cancers. <

\section{Les anticorps radiomarqués pour le traitement des cancers}

Jacques Barbet, Jean-François Chatal, Françoise Kraeber-Bodéré
Centre de recherche en cancérologie de Nantes-Angers, Université de Nantes, Inserm, U892, Institut de biologie, 9 , quai Moncousu, 44000 Nantes, France. Jacques.Barbet @nantes.inserm.fr

Gy) reçue par les tumeurs sera donc plus grande et doit pouvoir les détruire alors que les tissus sains seront épargnés. Contrairement à la radiothérapie externe qui administre des rayonnements électromagnétiques ou des particules de haute énergie émises par des sources radioactives ou des accélérateurs sur des lésions tumorales localisées par imagerie, la RIT vise des tumeurs disséminées, accessibles à l'anticorps marqué après son injection, le plus souvent par voie intraveineuse.

II y a peu de différence de principe entre RIT et anti corps «armés » à l'aide de composés chimiothérapeutiques ou de toxines $(\rightarrow)$, si ce n'est que la radioactivité est plus toxique

$(\rightarrow)$ voir J.F. Haeuw des anticorps marqués à l'iode 131 [2]. Ce tout premier patient, atteint de mélanome métastatique, a bénéficié d'une réponse spectaculaire, mais les essais suivants n'ont pas eu un résultat aussi heureux. Cinquante ans plus tard, l'application thérapeutique des anticorps vecteurs de radioactivité (radio-immunothérapie: RIT) a abouti à la mise sur le marché de deux anticorps marqués, l'un avec l'iode 131, l'autre avec l'yttrium 90 (Figure I), tous deux ciblant l'antigène CD20 et le lymphome malin non hodgkinien de $(\rightarrow)$ voir G. Cartron et phénotype $B$ [3] $(\rightarrow)$.

J.F. Rossi, p. 1085

Le concept à la base de la RIT est simple: un anticorps rencontrant une cellule tumorale exprimant l'antigène cible a une certaine probabilité de s'y fixer. Le temps de résidence de l'activité transportée par cet anticorps est donc plus long dans la tumeur que dans les autres tissus. La dose d'irradiation (quantité d'énergie déposée par masse de tissus, exprimée en joules/kg ou grays, qu'à une distance qui correspond aux parcours des particules dans la matière vivante (de la centaine de microns au centimètre, Tableau 1). Cette action à distance produit un phénomène de feu croisé absent dans les autres approches. Le couplage d'un atome radioactif à une molécule d'anticorps, chimérique ou humanisé pour éviter d'immuniser les patients [4], ne pose en général pas de problème (Figure I) et on dispose d'une gamme d'atomes radioactifs (Tableau I). De plus, I'internalisation de l'anticorps par la cellule cible n'est pas nécessaire à l'activité, mais n'est pas non plus un problème, car les métaux radioactifs restent piégés dans les cellules.

Les questions qui se posent aujourd'hui ne concernent donc plus la faisabilité clinique de la RIT, mais plutôt 


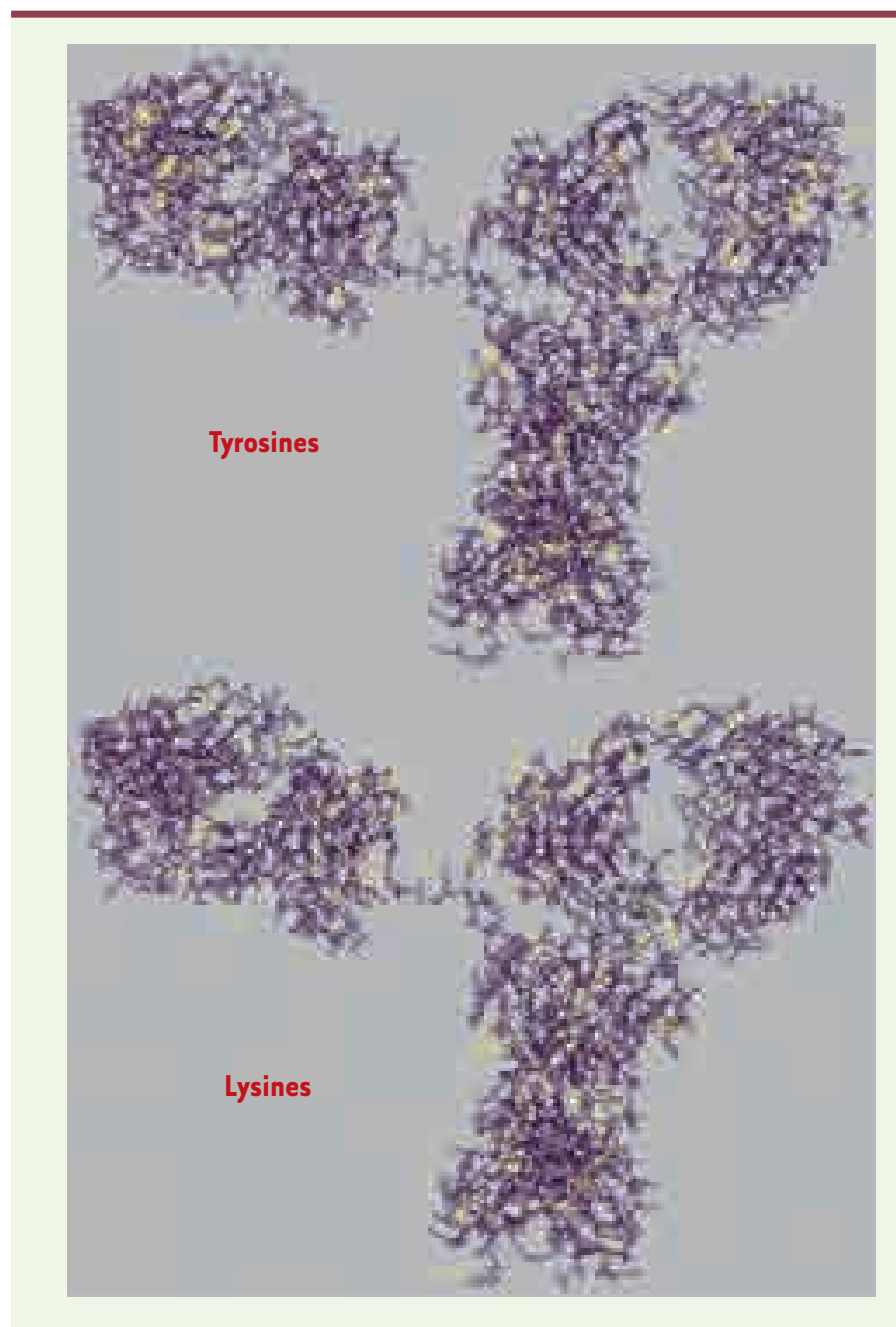

Figure 1. Structure cristallographique d'un anticorps (immunoglobuline G). Cette structure a été établie par Saphire et collègues [42] et représentée à l'aide du logiciel Cn3D version 4.1. Les fragments Fab de cet anticorps sont reconnaissables dans la partie supérieure des images. Les tyrosines (haut) et les lysines (bas) sont colorées en jaune pour montrer leur distribution dans l'ensemble de la molécule. On remarque une répartition assez uniforme de ces résidus à l'exception d'une concentration de tyrosines au voisinage des sites de liaison anticorps (extrémités supérieures droite et gauche). Ces tyrosines sont plutôt enfouies dans la structure. Cette répartition est très généralement retrouvée dans les anticorps. Dans la plupart des cas, il s'avère que l'on peut les marquer avec de l'iode radioactif par oxydation de l'iode (chloramine T, lodogen) et réaction directe avec les tyrosines de l'anticorps sans perte de réactivité. On peut également modifier ces molécules avec des agents chélatants bifonctionnels sous forme d'esters activés ( $\mathrm{N}$-hydroxysuccinimide) ou d'isothiocyanates qui réagissent avec les lysines pour un marquage avec des atomes radioactifs métalliques. Les lysines sont aussi utilisées pour le marquage avec des réactifs marqués à l'astate 211 (ester de $\mathrm{N}$-hydroxy-succinimide de l'acide m-astato-benzoïque).

son extension à d'autres pathologies que les lymphomes [4]. En effet, il faut reconnaître que l'efficacité thérapeutique de la RIT n'est pas encore démontrée dans les tumeurs solides ni dans d'autres hémopa- thies malignes comme les leucémies aiguës ou le myélome multiple. Dans cette revue, quelques avancées et voies de recherche seront décrites en partant de situations cliniques particulières. Nous verrons que, suivant que la maladie se présente comme une tumeur constituée ou au contraire comme un ensemble de cellules dispersées, les solutions envisageables ne sont pas les mêmes. Nous verrons également que, sauf exception, la RIT a peu de chances de venir à bout de cancers avancés lorsque les masses tumorales sont importantes. La RIT est proposée aujourd'hui dans des contextes de tumeurs minimales ou en présence de masses résiduelles ou en traitement adjuvant. Des traitements multimodalités qui sont la règle dans les autres approches thérapeutiques, mais qui n'ont pas encore été vraiment mis en œuvre avec la RIT, devraient suivre. Nous verrons aussi que la RIT semble plus à même d'augmenter la survie des malades que d'induire des réponses cliniques évaluables avec des critères morphologiques de type $\mathrm{RECIST}^{1}$. Ce qui d'ailleurs est un paradoxe qui montre que cette approche est beaucoup moins simple que ce que laisse supposer son concept de base.

\section{Les lymphomes}

Les lymphomes malins non hodgkiniens de phénotype $B$ comprennent de nombreux sous-types classés en fonction de leur évolution agressive ou indolente. Le lymphome folliculaire est la forme la plus fréquente des lymphomes B indolents. Si le lymphome folliculaire est une maladie d'évolution lente, il reste encore pratiquement toujours fatal dans sa forme disséminée. Alors qu'aucun progrès thérapeutique n'avait été réalisé entre 1960 et 1996 [5], aujourd'hui le traitement par rituximab (anticorps anti-CD20) combiné à la chimiothérapie induit des taux de réponse de 70 à $100 \%$, sans toutefois obtenir de guérison (les réponses durent de 18 à 50 mois) [6] $(\rightarrow)$. Les anticorps $(\rightarrow)$ voir G. Cartron et vecteurs de radioactivité, ${ }^{90} \mathrm{y}$-ibri- J.F. Rossi, page 1085 tumomab tiuxétan (Zévalin ${ }^{\circledR}$ ) et ${ }^{131}$-tositumomab $\left(\right.$ Bexxar $\left.{ }^{\circledR}\right)$, tous deux dérivés d'anticorps anti-CD20 et combinant RIT et injection de fortes doses de rituximab pour le premier et de tositumomab pour le second (voir ci-dessous), montrent des efficacités supérieures en termes de taux de réponse à celle des anticorps utilisés

${ }^{1}$ RECIST (Response evaluation criteria in solid tumors) est un ensemble de règles qui établissent des critères basés sur la taille des lésions tumorales pour définir un cancer qui s'améliore (réponse), ne bouge pas (stable), ou s'aggrave (progression) pendant le traitement. Ces critères ont été élaborés et publiés initialement en 2000 conjointement par l'EORTC (European organization for research and treatment of cancer), le $\mathrm{NCl}$ (National Cancer Institute) aux États-Unis, et le National Cancer Institute of Canada clinical trials group. La pertinence de ces critères RECIST lors de l'utilisation des biothérapies est aujourd'hui discutée. 


\begin{tabular}{|c|c|c|c|c|c|c|c|c|}
\hline & $\begin{array}{c}T_{1 / 2} \\
(\text { heures) } \\
\alpha\end{array}$ & $\begin{array}{l}\text { Émis- } \\
\text { sion' }^{b}\end{array}$ & $\begin{array}{l}\varepsilon \max \\
(\mathrm{keV})^{c}\end{array}$ & $\begin{array}{l}\text { Parcours } \\
(\mathrm{mm})^{\mathrm{c}}\end{array}$ & Marquage & Vecteurs & Avantages & Inconvénients \\
\hline lode 131 & 193 & $\begin{array}{l}\beta \\
\gamma\end{array}$ & $\begin{array}{l}610 \\
362\end{array}$ & 2,9 & - Direct & $\begin{array}{l}\text { - Seul (cancer } \\
\text { de la thyroïde, } \\
\text { imagerie et } \\
\text { thérapeutique) } \\
\text { - Anticorps }\end{array}$ & $\begin{array}{l}\text { - Faible coût } \\
\text { - Énergie faible } \\
\text { - Longue } \\
\text { demi-vie }\end{array}$ & $\begin{array}{c}\text { - Gamma de haute } \\
\text { énergie } \\
\text { - Instabilité } \\
\text { du marquage } \\
\text { si internalisation }\end{array}$ \\
\hline yttrium 90 & 64 & $\beta$ & 2250 & 11 & $\begin{array}{l}\text { - Chélatants } \\
\text { polyamino-car- } \\
\text { boxyliques : DOTA }\end{array}$ & $\begin{array}{l}\text { - Peptides } \\
\text { - Anticorps }\end{array}$ & - Commercial & $\begin{array}{c}\text { - Énergie élevée } \\
\text { - Pas de rayonnement } \\
\text { gamma }\end{array}$ \\
\hline Rhénium 188 & 17 & $\begin{array}{l}\beta \\
\gamma\end{array}$ & $\begin{array}{c}2120 \\
155\end{array}$ & 10 & $\begin{array}{l}\text { - Direct } \\
\text { - Nombreux } \\
\text { complexes N2S2 ou } \\
\text { N3S (chimie analo- } \\
\text { gue à celle du } \\
\text { technétium) }\end{array}$ & $\begin{array}{l}\text { - Lipiodol } \\
\text { - Anticorps }\end{array}$ & $\begin{array}{l}\text { - Disponible } \\
\text { sous forme de } \\
\text { générateur } \\
\text { - Imagerie } \\
\text { gamma }\end{array}$ & $\begin{array}{l}\text { - Énergie élevée } \\
\text { - Chimie complexe } \\
\text { - Demi-vie courte }\end{array}$ \\
\hline Lutétium 177 & 161 & $\begin{array}{l}\beta \\
\gamma\end{array}$ & $\begin{array}{l}498 \\
208\end{array}$ & 2 & $\begin{array}{l}\text { - Chélatants poly- } \\
\text { amino-carboxyli- } \\
\text { ques: DOTA }\end{array}$ & $\begin{array}{l}\text { - Peptides } \\
\text { - Anticorps }\end{array}$ & $\begin{array}{l}\text { - Énergie faible } \\
\text { - Demi-vie } \\
\text { longue }\end{array}$ & \\
\hline Cuivre 67 & 59 & $\begin{array}{l}\beta \\
\gamma\end{array}$ & $\begin{array}{c}395-577 \\
184\end{array}$ & 1,8 & $\begin{array}{c}\text { - Nombreux agents } \\
\text { complexants }\end{array}$ & $\begin{array}{l}\text { - Peptides } \\
\text { - Anticorps }\end{array}$ & $\begin{array}{l}\text { - Énergie faible } \\
\text { - Demi-vie } \\
\text { intermédiaire } \\
\text { - Métabolisme } \\
\text { favorable }\end{array}$ & $\begin{array}{l}\text { - Très difficile } \\
\text { à produire (cyclotrons } \\
\text { de haute énergie } \\
\text { et de haute intensité) }\end{array}$ \\
\hline
\end{tabular}

Tableau I. Principaux radionucléides utilisés en radio-immunothérapie. ${ }^{a}$ Le temps de demi-vie du radionucléide doit être rapproché du temps de demi-vie de son vecteur ou plus précisément du temps de résidence dans la tumeur. Avec un temps de demi-vie très court (bismuth 213), une grande partie des atomes radioactifs seront désintégrés avant d'avoir atteint leur cible. Avec une demi-vie longue (iode 131, lutétium 177), il faut s'assurer que le temps de résidence dans la tumeur est du même ordre. ${ }^{b}$ Seules les émissions de particules massives ( $\alpha$, $\beta$, électrons Auger) délivrent localement assez d'énergie pour un usage thérapeutique. Une émission $\gamma$ ou $X$ supplémentaire permet de suivre par imagerie la distribution de la radioactivité et d'effectuer une dosimétrie, mais les rayonnements $\gamma$ de très haute énergie (iode 131) donnent de mauvaises images et posent des problèmes de radioprotection. ${ }^{c} L^{\prime}$ 'énergie des particules émises conditionne leur parcours et leur transfert linéique d'énergie (TEL). Un parcours de l'ordre de la taille des lésions est évidemment préférable pour que le dépôt d'énergie, l'irradiation, concerne majoritairement les cellules cibles.

seuls [3]. Le traitement avec des doses myéloablatives de ${ }^{131} \mid$-tositumomab est très efficace, au prix d'une greffe de cellules souches hématopoïétiques (moelle osseuse) autologues et des risques associés [7]. L'association de Zévalin à la chimiothérapie intensive avant autogreffe est potentiellement plus efficace que la RIT à dose conventionnelle, mais nécessite une dosimétrie précise pour éviter les effets toxiques, notamment lors de l'administration de Zévalin à haute activité [8]. Le ${ }^{90}$-ibritumomab tiuxetan en consolidation après traitement conventionnel allonge la durée de survie sans progression de 13,5 à 37 mois $(p<0,001)[9,10]$, ce qui a permis d'étendre récemment l'indication de Zévalin à la consolidation du traitement de première ligne. Dans les autres types histologiques, lymphomes agressifs, la RIT avec des anticorps anti-CD20 et d'autres anticorps (anti-CD22, par exemple) montre aussi une efficacité qui devra encore être confirmée par des études randomisées [11]. Pour traiter ces 
lymphomes agressifs, les approches «haute dose», combinées avec l'autogreffe, type Z-BEAM², sont privilégiées [7].

Parmi les raisons évoquées de l'efficacité des anticorps radiomarqués dans le lymphome, la synergie entre l'anticorps nu, qui est injecté en masse (250 $\mathrm{mg} / \mathrm{m}^{2}$ de rituximab), et l'anticorps marqué, le ${ }^{90} y$-ibritumomab tiuxetan injecté quatre heures après ${ }^{3}$, a été considérée comme indispensable pour obtenir un effet à long terme sur la tumeur [12]. Toutefois des essais en cours avec l'épratuzumab (anti-CD22) marqué à l'yttrium-90 montrent une efficacité au moins équivalente avec une dose totale d'anticorps plus faible $(0,75$ ou $1,5 \mathrm{mg} / \mathrm{kg}$ suivant les protocoles) injectée en une seule fois [11]. En revanche, il n'y a pas de corrélation entre la dose d'irradiation absorbée par la tumeur et la réponse, ce qui peut s'expliquer par la difficulté d'estimer correctement les doses absorbées (imagerie monophotonique avec l'indium 111 en place de l'yttrium-90), mais pourrait suggérer que des mécanismes autres que la radioactivité sont à l'œuvre. Malgré tout, la perméabilité des tumeurs donnant une bonne accessibilité à l'anticorps et la radiosensibilité des lymphomes (une irradiation corporelle totale de quelques Gy a un effet tumoricide pour les leucémies et les lymphomes [13]) restent les principales raisons de l'efficacité de la RIT dans le lymphome.

Aujourd'hui les recherches s'orientent surtout vers de nouveaux modes d'utilisation: I'introduction de la RIT à un stade précoce de la maladie, la combinaison des traitements, le fractionnement et l'utilisation en mode adjuvant, ou l'utilisation du préciblage qui a été évalué au stade préclinique, et moins sur l'amélioration des anticorps ou des radionucléides $[14,15]$. Pour ces pathologies, des anticorps marqués de façon conventionnelle soit avec de l'iode 131 ( ${ }^{131}$ I-tositumomab), soit avec de l'yttrium 90 après modification de l'anticorps par un agent chélatant couplé à l'anticorps (un dérivé activé du benzyl-méthyl-DTPA pour ${ }^{90} y$-ibritumomab tiuxetan et le DOTA pour l'épratuzumab) semblent suffisants (Figure 1). Les radionucléides sont également conventionnels. L'iode 131 est utilisé depuis plus de cinquante ans dans le cancer de la thyroïde et l'yttrium 90 est commercialisé aujourd'hui sous le nom d'ytracis (Tableau I). Ces radionucléides ne sont pourtant pas idéaux pour la RIT. L'iode 131 émet un rayonnement gamma qui impose, en Europe, un confinement des malades en chambres radioprotégées pendant plusieurs jours. Pour cette raison le ${ }^{131}$ I-tositumomab n'est commercialisé qu'aux États-Unis. L'yttrium 90, lui, n'émet pas de rayonnement gamma, ce qui impose une substitution par un autre métal pour la dosimétrie à défaut d'yttrium 86 , émetteur de positons qui n'est produit qu'à la demande et est peu disponible.

\footnotetext{
${ }^{2}$ Chimiothérapie de type BEAM : carmustine, étoposide, aracytine et melphalan (la veille de la greffe autologue). $Z$ = Zévalin.

${ }^{3}$ Avant le traitement par RIT (zévalin), les patients reçoivent une injection de rituximab destinée à éliminer les lymphocytes $B$ de la circulation et à bloquer une partie des sites de liaison sur les lymphocytes malins présents dans la rate. Le Zevalin est administré secondairement et la dose de charge d'anticorps non marqué a pour but de lui permettre d'irradier plus spécifiquement les lymphocytes malins présents dans les autres tissus. Au moment des premiers essais cliniques, l'injection de cette dose de charge était tout à fait justifiée par les masses tumorales élevées des malades inclus dans les essais cliniques, dont certains présentaient des splénomégalies importantes. Aujourd'hui, les patients traités sont différents et, particulièrement en situation de traitement de consolidation, l'intérêt de l'administration de cette dose élevée d'anticorps non marqué dans le traitement standard au Zévalin devrait être réévalué.
}

\section{Les tumeurs solides}

Comment améliorer l'irradiation des tumeurs solides L'irradiation externe requiert plus de 60 Gy pour stériliser une tumeur solide. Dans ces conditions on peut s'attendre à ce qu'il y ait des difficultés à utiliser la RIT contre les tumeurs solides. Chez l'animal pourtant, les grands précurseurs dans ce domaine, David Goldenberg aux États-Unis et Jean-Pierre Mach en Suisse, ont montré depuis longtemps déjà des résultats encourageants dans diverses tumeurs humaines greffées chez la souris nude [16-18]. Chez l'homme, de nombreux essais cliniques, généralement de phase I ou II qui incluent des petits effectifs de patients présentant des tumeurs avancées, ont donné des résultats médiocres, même avec des anticorps ciblant très efficacement les tumeurs [19]. Les taux de réponse clinique sont considérés comme faibles, même s'ils ne le sont pas plus qu'avec la chimiothérapie. Le critère de stabilisation de la maladie, qui est aujourd'hui pris en compte dans le développement clinique de certains inhibiteurs de tyrosine-kinases (voir par exemple le cas du sorafenib ${ }^{4}$ ) [20], n’a généralement pas été considéré pour la RIT [21]. Les résultats cliniques de la RIT mériteraient d'être reconsidérés dans ce sens, comme nous le verrons plus loin.

Augmenter l'irradiation des tumeurs peut passer par une amélioration des vecteurs. Des anticorps possédant une meilleure affinité ont montré une activité antitumorale accrue [22], et une constante de dissociation de l'ordre du nanomolaire ( $10^{-9}$ mole par litre) semble suffisante. Mais cette voie n'a pas été systématiquement suivie. La réduction de la taille du vecteur, en utilisant divers fragments d'anticorps, $\left.\mathrm{F}(\mathrm{ab})_{2}\right)_{2}$, Fab ou constructions à base de scFv $(\rightarrow)$, n'a pas non plus apporté de résultat déci- $\quad(\rightarrow)$ voir P. Chames et sif [23]. La diffusion accrue des peti-

D. Baty, page 1159

tes molécules dans l'organisme est contrebalancée par leur élimination plus rapide, ce qui est un avantage pour l'imagerie mais pas nécessairement pour la thérapie. La réduction de la toxicité hématologique des anticorps est compensée par une augmentation de la toxicité rénale. Les «affibodies » $\rightarrow$ ( $)$, constructions moléculaires combinatoires basées sur une

$\rightarrow$ voir T. Wurch et al., page 1169 protéine bactérienne extrêmement stable, la protéine $A$ du staphylocoque doré [24], n'échappent pas à cette règle [25], pas plus que les fragments d'anticorps ne comportant qu'une chaîne peptidique, préparés à partir des anticorps non classiques, dépourvus de chaîne

$\rightarrow$ voir P. Chames et légère, des camélidés [26] $(\rightarrow)$.

D. Baty, page 1159 dans certains types de cellules tumorales (BRAF, KIT et FLT- 3 ) et dans les vaisseaux sanguins tumoraux (CRAF, VEGFR-2, VEGFR-3 et PDGFR- $\beta$ ) 


\section{Techniques de préciblage}

Une approche différente a été proposée à la fin des années 1980. II s'agit d'un ensemble de techniques maintenant baptisées «préciblage». L'anticorps chargé de reconnaître la tumeur est couplé avec de l'avidine ou avec un anticorps antihaptène. Il est injecté, non marqué et c'est une petite molécule (dérivée de la biotine, très affine pour l'avidine, ou de l'haptène reconnu par le second anticorps), injectée plus tard, qui porte la radioactivité. Un tel système n'aurait pas d'intérêt s'il était utilisé tel quel. En fait il faut utiliser une astuce supplémentaire: chasser l'excès d'anticorps circulant avant l'injection de biotine radiomarquée par l'administration d'un composé spécifique biotinylé et glycosylé qui formera un complexe rapidement éliminé par le foie (système avidine-biotine) ou utiliser un vecteur de radioactivité se liant plus efficacement, grâce à un effet coopératif, sur les cellules tumorales qui ont fixé au préalable l'anticorps bispécifique qu'à l'anticorps bispécifique restant dans la circulation (haptènes bivalents de la technique AES). Ces techniques améliorent sensiblement l'efficacité du ciblage (Figure 2) et ont été appliquées avec un certain succès en RIT [27]. Une augmentation de la survie globale a même été observée chez des malades atteints de cancer médullaire de la thyroïde métastatique par rapport à un groupe contrôle historique de pronostic équivalent (110 versus 61 mois, $p<0,03)$ [28]. Il est à noter que cette augmentation de survie est obtenue par la stabilisation à long terme de la maladie, souvent sans réponse objective notable. Ces techniques sont maintenant envisagées dans de nombreuses indications, y compris en hématologie, mais elles restent difficiles à développer (production des anticorps bispécifiques, optimisation nécessaire des doses et délais d'administration des différents composants).

\section{Relation entre la taille des tumeurs \\ et la fixation de la radioactivité}

Un autre aspect de la question oriente aujourd'hui une grande partie de la recherche en RIT. La fixation tumorale de la radioactivité est une fonction décroissante de la taille des tumeurs. Les propriétés de vascularisation des tumeurs, avec une périphérie hypervascularisée et un centre hypoxique, voire nécrotique, et l'absence de percolation lymphatique, expliquent cette relation inverse $(\rightarrow)$. $(\rightarrow)$ voir G. Paintaud, Des essais dans des petites tumeurs et en situa- page 1057 tion adjuvante montrent des résultats très encourageants en termes de survie sans progression ou de survie globale [29-31]. Ainsi les résultats positifs obtenus dans le cancer de la prostate avec l'anticorps J591 «désimmunisé » [32] pourraient se voir confirmés chez des patients à risque, après exérèse de la prostate, qui recevraient la RIT au vu d'une élévation isolée du «prostate specific antigen » (PSA). Ce changement d'objectif clinique a entraîné une réflexion sur le choix du radionucléide (Tableau I). Le lutétium 177, émetteur $\beta^{-}$de faible énergie, est aujourd'hui préféré à l'yttrium 90 pour traiter des petites lésions tumorales [33]. Le cuivre 67 est une autre option [34] qui pourra se développer grâce à de nouveaux moyens de production (cyclotrons de haute énergie et haute intensité comme Arronax, qui vient d'être installé à Nantes).

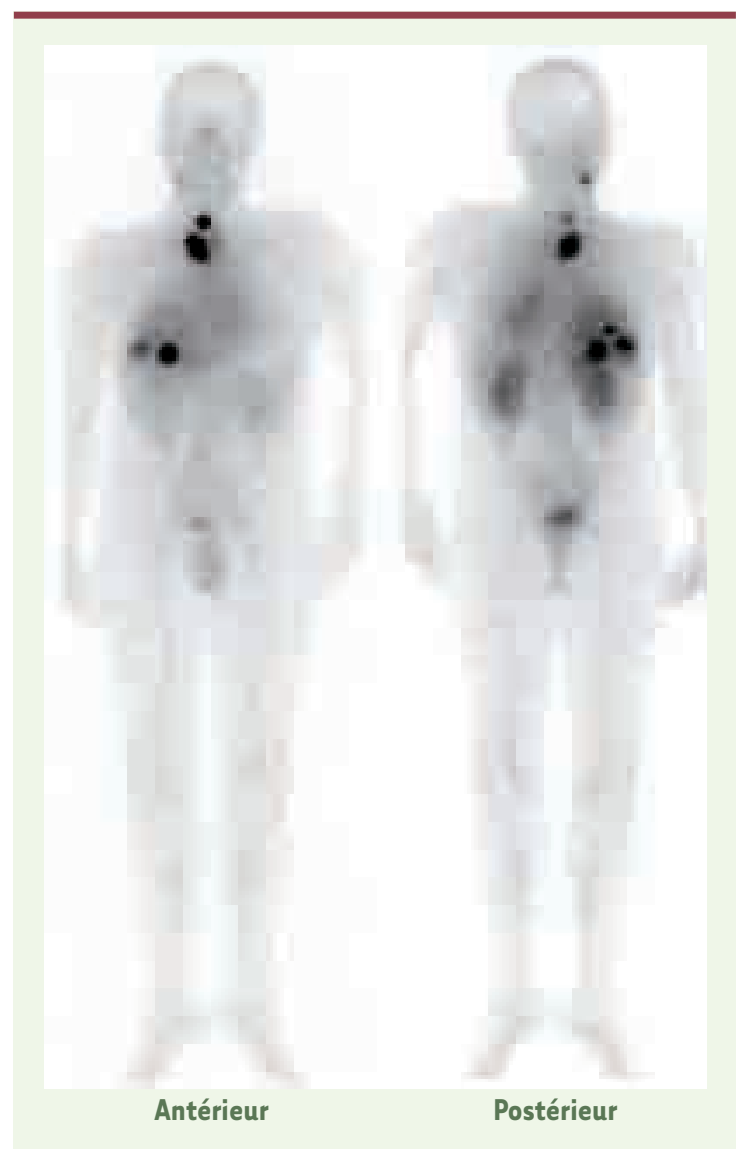

Figure 2. Immunoscintigraphie corps entier d'un patient présentant de multiples métastases de cancer médullaire de la thyroïde. Les deux images, antérieure et postérieure, révèlent les multiples métastases délimitées avec un fort contraste grâce à une technique de préciblage (AES) ciblant l'antigène carcinoembryonnaire [43]. De telles images, qui ne sont pas exceptionnelles, démontrent l'efficacité du ciblage de radioactivité par les anticorps. Malgré cela, l'efficacité de la RIT dans les tumeurs solides reste généralement insuffisante car il est difficile d'obtenir un aussi bon contraste de la tumeur par rapport aux tissus sains lorsqu'une dose potentiellement tumoricide, donc beaucoup plus élevée, est utilisée. Par ailleurs, ces images scintigraphiques, enregistrées à plusieurs temps du traitement, permettent de calculer les doses absorbées par les tumeurs et les différents organes, ce qui donne une très bonne sécurité à la détermination des doses maximales tolérées en RIT [44].

\section{Les leucémies et le myélome multiple}

Dans ces pathologies, les cellules tumorales sont disséminées. Les anticorps anti-CD33, anti-CD45 ou anti-CD66 marqués avec l'iode 131, l'yttrium 90 ou le rhénium 188 diminuent le risque de rechute après transplantation de moelle osseuse ${ }^{5}$ dans la leucémie

${ }^{5}$ Donneurs compatibles, avec ou sans lien de parenté. 
myéloïde aigüe [35]. Le mylotarg, un anticorps anti-CD33 armé par une toxine, la calichéamycine, est commercialisé pour le traitement de la leucémie aigüe myéloïde [36], mais la RIT seule n'a pas démontré une grande efficacité $(\rightarrow)$. Une $(\rightarrow)$ voir G. Cartron et explication peut être avancée. Les radionucléi- J.F. Rossi, page 1085 des déposent leur énergie dans un volume qui dépend de la nature de la particule émise et de son énergie. Ainsi les rayons $\gamma$ ne sont que partiellement atténués en traversant le corps humain, ce qui les rend utiles en imagerie mais pas en thérapeutique. Les rayonnements $\beta^{-}$, qui sont en fait des électrons, déposent leur énergie (quelques centaines de keV à quelques MeV) sur une distance variant du millimètre au centimètre (transfert d'énergie linéique ou TEL faible). On comprend aisément que si un atome émetteur $\beta^{-}$est fixé sur une cellule tumorale isolée, la plus grande partie de l'énergie de la particule émise sera délivrée au-delà de la cellule tumorale elle-même, voire même dans les tissus sains environnants. L'irradiation aura raté sa cible et n'aura qu'une très faible efficacité [3].

Les rayonnements $\alpha$, constitués de noyaux d'hélium, ont eux un TEL beaucoup plus élevé et un parcours plus court ( $0,1 \mathrm{~mm}$ environ). Ils ont de plus une énergie élevée (plusieurs MeV). À activité égale, ils sont beaucoup plus efficaces que les rayonnements $\beta^{-}$pour irradier des cellules isolées [37]. Les radionucléides émetteurs d'électrons Auger ${ }^{6}$ déposent leur énergie sur une distance de quelques microns, la question ouverte dans ce cas étant celle de la localisation subcellulaire optimale (noyau, cytoplasme, membrane) du radionucléide pour une efficacité maximale [38]. Les fortes activités nécessaires dans ce cas peuvent poser des problèmes de radioprotection. Pour ce qui est des émetteurs $\alpha$, le bismuth 213 obtenu à partir de générateurs d'actinium 225 et l'astate 211 produit en cyclotron sont les plus utilisés. Leur toxicité proscrit des doses élevées. Dans ce cas, ils ne peuvent être envisagés que dans des situations cliniques de très faible masse tumorale, traitement adjuvant, métastases microscopiques, tumeur résiduelle. Ces situations sont aussi celles où les cellules tumorales sont isolées ou en micro-agrégats, donc celles où I'utilisation de rayonnement à fort TEL est indispensable. Des essais cliniques ont eu lieu. Ils ont montré la faisabilité du traitement de la leucémie aigüe myéloïde avec un anticorps anti-CD33 marqué au bismuth 213, mais ils ont montré aussi que, à un stade avancé de la maladie, le nombre de cellules tumorales pouvait être de l'ordre du nombre d'atomes radioactifs administrés [39]. Un traitement de debulking est donc nécessaire. Ces émetteurs de particules $\alpha$ sont également envisagés pour traiter la tumeur résiduelle par administration loco régionale (tumeurs cérébrales, de l'ovaire, de la vessie, carcinoses péritonéales). Les essais précliniques, et les rares essais cliniques sont prometteurs [40].

${ }^{6}$ L'émission des électrons Auger provient de la désexcitation d'atomes par éjection d'un de leurs électrons de cœur. Ce phénomène apparaît lors des désintégrations ou conversions nucléaires qui laissent la couronne électronique des atomes dans un état excité. Ces électrons Auger ont des énergies faibles (typiquement 10 à $20 \mathrm{keV}$ ), mais cette énergie est déposée dans un très petit volume (quelques microns de diamètre). Les électrons Auger ont donc un potentiel thérapeutique.

\section{Conclusions}

La RIT a fait ses preuves dans le traitement du lymphome et devrait bientôt proposer des nouvelles options thérapeutiques pour d'autres tumeurs. Son domaine d'application privilégié devrait être des situations de maladies minimales, et elle devrait être utilisée en traitement de consolidation ou en traitement adjuvant. Notons que jusqu'à récemment, les essais cliniques ont été menés avec de faibles moyens (essais académiques ou promus par des sociétés radiopharmaceutiques qui ne disposent pas des moyens des grandes compagnies). Le plus souvent, une seule administration d'un seul composé thérapeutique a été testée. Dans ces conditions, on pouvait espérer au mieux obtenir des indices d'efficacité qu'il convient aujourd'hui de concrétiser dans le cadre d'approches multimodalités. Comme pour les médicaments cytostatiques, c'est sans doute en évaluant directement l'effet du traitement sur la survie des malades que l'on pourra se convaincre de l'intérêt de la RIT. Techniquement, des nouvelles approches comme le préciblage et de nouveaux radionucléides à fort TEL (émetteurs $\alpha$ ou d'électrons Auger) devraient être à même de produire de réelles avancées en apportant des solutions pour l'éradication des cellules souches tumorales, d'autant que les effets néfastes à long terme (notamment induction de myélodysplasies et de leucémies secondaires) ne semblent pas plus fréquents qu'avec la chimiothérapie [41]. $\diamond$

\section{SUMMARY}

Radiolabeled antibodies for cancer treatment

The first treatment ever by radio-immunotherapy (RIT) was performed by William H. Beierwaltes in 1951 and was a success. Fifty years later, the main question is to find ways of extending the success of radiolabelled antiCD20 antibodies in indolent non-Hodgkin's lymphoma to other forms of cancer. Solid tumours are much more radioresistant than lymphomas, but they respond to RIT if the lesions are small. Clinical situations of residual or minimal disease are thus the most likely to benefit from RIT in the adjuvant or consolidation settings. For disseminated disease, like leukemias or myelomas, the problem is different: beta ${ }^{-}$particles emitted by the radioactive atoms classically used for cancer treatment (iodine-131 or yttrium-90) disperse their energy in large volumes (ranges $1 \mathrm{~mm}$ to $1 \mathrm{~cm}$ ) and are not very effective against isolated cells. Advances in RIT progress in two directions. One is the development of pretargeting strategies in which the antibody is not labelled but used to provide binding sites to small molecular weight radioactivity vectors (biotin, haptens). These techniques have 
been shown to increase tumour to non-target uptake ratios and anti-tumour efficacy has been demonstrated in the clinic. The other approach is the use of radionuclides adapted to the various clinical situations. Lutetium-177 or copper-67, because of the lower energy of their emission, their relatively long half-life and good gamma emission, may significantly improve RIT efficacy and acceptability. Beyond that, radionuclides emitting particles such as alpha particles or Auger electrons, much more efficient to kill isolated tumour cells, are being tested for RIT in the clinic. Finally, RIT should be integrated with other cancer treatment approaches in multimodality protocols. Thus RIT, now a mature technology, should enter a phase of well designed and focused clinical developments that may be expected to afford significant therapeutic advances. $\diamond$

\section{CONFLIT D'INTÉRÊTS}

Les auteurs déclarent n'avoir aucun conflit d'intérêts concernant les données publiées dans cet article.

\section{RÉFÉRENCES}

1. Sisson J, Shapiro B, Beierwaltes WH, et al. Treatment of malignant pheochromocytoma with a new radiopharmaceutical. Trans Assoc Am Physicians $1983 ; 96: 209-17$.

2. Suresh C, Srivastava L. Investigators review potential of antibodies in cancer detection and therapy. J Nucl Med $1987 ; 28: 143-7$.

3. Buchegger F, Press OW, Delaloye AB, Ketterer N. Radiolabeled and native antibodies and the prospect of cure of follicular lymphoma. Oncologist 2008; $13: 657-67$.

4. Bellet D, Dangles-Marie V. Anticorps humanisés en thérapeutique. Med Sci (Paris) $2005 ; 21: 1054-62$

5. DeNardo GL, DeNardo SJ, Balhorn R. Systemic radiotherapy can cure lymphoma: a paradigm for other malignancies? Cancer Biother Radiopharm $2008 ; 23: 383-97$

6. Horning SJ. Natural history of and therapy for the indolent non-Hodgkin's lymphomas. Semin Oncol 1993; $20: 75-88$.

7. Cheson BD, Leonard JP. Monoclonal antibody therapy for B-cell non-Hodgkin's lymphoma. N Engl J Med 2008 ; 359 : 613-26.

8. Park $\mathrm{SI}$, Press $\mathrm{OW}$. Radioimmunotherapy for treatment of B-cell lymphomas and other hematologic malignancies. Curr Opin Hematol 2007 ; 14 : 632-8.

9. Zhang MM, Gopal AK. Radioimmunotherapy-based conditioning regimens for stem cell transplantation. Semin Hematol 2008 ; 45 : 118-25.

10. Chatal JF, Kraeber-Bodéré F, Barbet J. Consolidation radioimmunotherapy of follicular lymphoma: a step towards cure? Eur J Nucl Med Mol Imaging 2008 $35: 1236-9$.

11. Morschhauser F, Radford J, Van Hoof A, et al. Phase III trial of consolidation therapy with yttrium-90-ibritumomab tiuxetan compared with no additional therapy after first remission in advanced follicular lymphoma. J Clin Oncol $2008 ; 26: 5156-64$.

12. Bodet-Milin C, Kraeber-Bodéré F, Dupas B, et al. Evaluation of response to fractionated radioimmunotherapy with ${ }^{90} \mathrm{Y}$-epratuzumab in non-Hodgkin's lymphoma by ${ }^{18} \mathrm{~F}$-fluorodeoxyglucose positron emission tomography. Haematologica $2008 ; 93: 390-7$.

13. Ivanov A, Swann R, Illidge T. New insights into the mechanisms of action of radioimmunotherapy in lymphoma.J Pharm Pharmacol 2008; $60: 987-98$.

14. Wheldon TE. The radiobiological basis of total body irradiation. BrJ Radiol $1997 ; 70: 1204-7$.

15. Sharkey RM, Karacay H, Chang CH, et al. Improved therapy of non-Hodgkin's lymphoma xenografts using radionuclides pretargeted with a new anti-CD20 bispecific antibody. Leukemia 2005 ; 19 : 1064-9.

16. Press OW, Corcoran M, Subbiah K, et al. A comparative evaluation of conventional and pretargeted radioimmunotherapy of $\mathrm{CD} 20$-expressing lymphoma xenografts. Blood $2001 ; 98: 2535-43$.
17. Goldenberg DM, Gaffar SA, Bennett SJ, Beach JL. Experimental radioimmunotherapy of a xenografted human colonic tumor (GW-39) producing carcinoembryonic antigen. Cancer Res 1981; $41: 4354-60$.

18. Sharkey RM, Pykett MJ, Siegel JA, et al. Radioimmunotherapy of the GW-39 human colonic tumor xenograft with ${ }^{131}$ I-labeled murine monoclonal antibody to carcinoembryonic antigen. Cancer Res $1987 ; 47: 5672-7$.

19. Buchegger F, Vacca A, Carrel S, et al. Radioimmunotherapy of human colon carcinoma by ${ }^{131} \mid-$ labelled monoclonal anti-CEA antibodies in a nude mouse model. Int J Cancer 1988; 41:127-34.

20. Sharkey RM, Goldenberg DM. Perspectives on cancer therapy with radiolabeled monoclonal antibodies. J Nucl Med $2005 ; 46:$ S115-27.

21. Hahn 0, Stadler W. Sorafenib. Curr Opin Oncol $2006 ; 18: 615-21$.

22. Brouwers AH, Mulders PF, de Mulder PH, et al. Lack of efficacy of two consecutive treatments of radioimmunotherapy with ${ }^{131} \mathrm{I}-\mathrm{cG} 250$ in patients with metastasized clear cell renal cell carcinoma. J Clin Oncol $2005 ; 23: 6540-8$.

23. Buchegger $F$, Pfister $C$, Fournier $K$, et al. Ablation of human colon carcinoma in nude mice by ${ }^{131} 1-$ labeled monoclonal anti-carcinoembryonic antigen antibody $\mathrm{F}\left(\mathrm{ab}^{\prime}\right)_{2}$ fragments. J Clin Invest 1989; $83: 1449-56$

24. Schlom J, Eggensperger D, Colcher D, et al. Therapeutic advantage of high-affinity anticarcinoma radioimmunoconjugates. Cancer Res $1992 ; 52: 1067-72$.

25. Nilsson Fy, Tolmachev V. Affibody molecules : new protein domains for molecular imaging and targeted tumor therapy. Curr Opin Drug Discov Dev $2007 ; 10$ : 167-75.

26. Muyldermans S. Single domain camel antibodies : current status. J Biotechnol $2001 ; 74: 277-302$.

27. Goldenberg DM, Sharkey RM, Paganelli G, et al. Antibody pretargeting advances cancer radioimmunodetection and radioimmunotherapy. J Clin Oncol $2006 ; 24: 823-34$.

28. Chatal JF, Campion L, Kraeber-Bodéré F, et al. Survival improvement in patients with medullary thyroid carcinoma who undergo pretargeted anti-carcinoembryonic-antigen radioimmunotherapy: a collaborative study with the French Endocrine Tumor Group. J Clin Oncol 2006 ; 24 : 1705-11.

29. Behr TM, Liersch T, Greiner-Bechert L, et al. Radioimmunotherapy of small-volume disease of metastatic colorectal cancer. Cancer $2002 ; 94: 1373-81$.

30. Liersch T, Meller J, Bittrich M, et al. Update of carcinoembryonic antigen radioimmunotherapy with ${ }^{131}$ I-labetuzumab after salvage resection of colorectal liver metastases : comparison of outcome to a contemporaneous control group. Ann Surg Oncol 2007; $14: 2577-90$.

31. Ychou M, Azria D, Menkarios C, et al. Adjuvant radioimmunotherapy trial with iodine-131-labeled anti-carcinoembryonic antigen monoclonal antibody F6 F(ab') ${ }_{2}$ after resection of liver metastases from colorectal cancer. Clin Cancer Res 2008 ; 14 : 3487-93.

32. Milowsky MI, Nanus DM, Kostakoglu L, et al. Phase I trial of yttrium-90-labeled anti-prostatespecific membrane antigen monoclonal antibody $J 591$ for androgen-independent prostate cancer.J Clin Oncol $2004 ; 22$ : 2522-31.

33. Bander NH, Milowsky MI, Nanus DM, et al. Phase I trial of ${ }^{177}$ lutetium-labeled J591, a monoclonal antibody to prostate-specific membrane antigen, in patients with androgen-independent prostate cancer. J Clin Oncol 2005; $23: 4591-601$.

34. Novak-Hofer I, Schubiger PA. Copper-67 as a therapeutic nuclide for radioimmunotherapy. Eur J Nucl Med Mol Imaging 2002; $29: 821-30$.

35. Kotzerke J, Bunjes D, Scheinberg DA. Radioimmunoconjugates in acute leukemia treatment : the future is radiant. Bone Marrow Transplant 2005 ; 36 : 1021-6.

36. Sievers $\varepsilon$ L, Appelbaum FR, Spielberger RT, et al. Selective ablation of acute myeloid leukemia using antibody-targeted chemotherapy : a phase I study of an anti-CD33 calicheamicin immunoconjugate. Blood 1999; 93 : 3678-84.

37. Supiot $S$, Faivre-Chauvet A, Couturier 0 , et al. Comparison of the biologic effects of MA5 and B-B4 monoclonal antibody labeled with iodine-131 and bismuth-213 on multiple myeloma. Cancer 2002 ; $94: 1202-9$.

38. Mattes MJ. Radionuclide-antibody conjugates for single-cell cytotoxicity. Cancer 2002 ; 94 : 1215 23.

39. Jurcic JG, Larson SM, Sgouros G, et al. Targeted alpha particle immunotherapy for myeloid leukemia. Blood 2002; $100: 1233-9$.

40. Zalutsky MR, Reardon DA, Akabani G, et al. Clinical experience with alpha-particle emitting 211At treatment of recurrent brain tumor patients with ${ }^{211} \mathrm{At}$-labeled chimeric antitenascin monoclonal antibody 81C6. J Nucl Med $2008 ; 49: 30-8$.

41. Roboz GJ, Bennett JM, Coleman M, et al. Therapy-related myelodysplastic syndrome and acute myeloid leukemia following initial treatment with chemotherapy plus radioimmunotherapy for indolent non-Hodgkin lymphoma. Leuk Res 2007 ; 31 : 1141-4.

42. Saphire $\varepsilon 0$, Parren PW, Pantophlet R, et al. Crystal structure of a neutralizing human IGG against HIV-1 : a template for vaccine design. Science $2001 ; 293$ : 1155-9

43. Barbet J, Peltier $P$, Bardet $S$, et al. Radioimmunodetection of medullary thyroid carcinoma using indium-111 bivalent hapten and anti-CEA $\mathrm{x}$ anti-DTPA-indium bispecific antibody. J Nucl Med 1998, 39, 1172-8.

44. Flux G, Bardies M, Monsieurs M, et al. The impact of PET and SPECT on dosimetry for targeted radionuclide therapy. $Z$ Med Phys $2006 ; 16: 47-59$. 